\title{
Potential effects of hormonal synchronized breeding on genetic evaluations of fertility traits in dairy cattle: A simulation study
}

\author{
G. A. Oliveira Junior, ${ }^{1 *}$ L. R. Schaeffer, ${ }^{1}$ F. Schenkel, ${ }^{1}$ F. Tiezzi, ${ }^{3}$ and C. F. Baes ${ }^{1,2}$ \\ ${ }^{1}$ Centre for Genetic Improvement of Livestock, Department of Animal Biosciences, University of Guelph, Guelph, ON, N1G-2W1, Canada \\ ${ }^{2}$ Institute of Genetics, Department of Clinical Research and Veterinary Public Health, University of Bern, Bern, 3001, Switzerland \\ ${ }^{3}$ Department of Animal Science, North Carolina State University, Raleigh 27695
}

\begin{abstract}
About $30 \%$ of producers use hormone protocols to synchronize ovulation and perform timed artificial insemination (AI) in Canada. Days from calving to first service (CTFS) and first service to conception (FSTC) become masked phenotypes leading to biased genetic evaluations of cows for these fertility traits. The objectives of this study were to (1) demonstrate and quantify the potential amount of bias in genetic evaluations, and (2) find a procedure that could remove the bias. Simulation was used for both objectives. The proposed solution was to identify cows that have been treated by hormone protocols, make their CTFS and FSTC missing, and perform a multiple trait analysis including traits that have high genetic correlations with CTFS and FSTC, and which are not affected by the hormone protocols themselves. A total of 12 scenarios (S1-S12) were tested, changing the percentage of herds and cows that were randomly selected to be under timed AI. Cows that were given hormone protocols had CTFS of $86 \mathrm{~d}$ and FSTC of 0, which were used in genetic evaluation. Four criteria were used to indirectly measure the presence of bias: (1) the correlation between true (TBV) and estimated (EBV) breeding values (accuracy); (2) the differences in the mean EBV of top 25, 50, and 75 sires; (3) changes in correlation between TBV and EBV rankings; and (4) the changes in mean EBV over the simulated generations. All criteria changed unfavorably and proportionally to the increased use of timed AI. The accuracy within each class of animals (cows, dams, or sires) decreased proportionally with increased use of timed AI, varying from 0.32 (S12) to 0.52 (S1) for bull EBV for CTFS. The average EBV of the top sires (best 25, 50, 75, or 100 sires) approached population average EBV values when increasing the number of treated animals. The sire rank correlation between
\end{abstract}

Received May 22, 2020.

Accepted November 15, 2020.

*Corresponding author: gerson@uoguelph.ca
EBV and TBV within simulated scenarios was smaller for scenarios with more synchronized animals, going from 0.38 (S12) to 0.67 (S1). The long-term use of hormonal synchronized cows clearly decreased the mean EBV over generations in the population for CTFS and FSTC. The inclusion of genetically correlated traits in a multiple trait model was effective in removing the bias due to the presence of hormonal synchronized cows. However, given the constraints within the simulation, it is important that further investigation with real data is conducted to determine the true effect of including timed AI records within genetic evaluations of fertility traits in dairy cattle.

Key words: bias, dairy cattle, fertility, genetics

\section{INTRODUCTION}

Reproductive efficiency of dairy cows, which directly affects the profitability of the dairy industry, has been a common concern among producers. Reproductive efficiency traits are generally reported to have low genetic heritability (less than 0.10) and negative genetic correlations with production traits (VanRaden et al., 2004; Jamrozik et al., 2005). Part of the observed residual variation for these traits can be attributed to nongenetic factors, such as management and environmental variation, as well as low quality phenotypic information (Miglior et al., 2017).

The success of the fertility performance of dairy farms, especially for AI programs, starts with estrus detection (Silper et al., 2017; Reith and Hoy, 2018). This task can be labor intensive and time consuming, with a high probability of errors, especially on large farms (Diskin and Sreenan, 2000; Colazo and Mapletoft, 2014). In addition, estrus expression has decreased on high-producing Holstein cows over the years, with up to $60 \%$ of ovulations accompanied by no standing mount (Butler, 2003; Kerbrat and Disenhaus, 2004; Roelofs et al., 2005). This dependency on estrus detection can be overcome by the use of hormonal protocols to synchronize follicle growth, corpus luteum regres- 
sion, and ovulation. This practice, termed timed AI, facilitates lactating cows to start a new estrous cycle, eliminating the need for detection of estrus (Roelofs et al., 2005; Dolecheck et al., 2016).

The use of timed AI varies among farms, where some producers might selectively synchronize cows with delayed or unobserved estrus, whereas others have a more intensive approach, synchronizing all cows (Goodling et al., 2005). As such, this practice can then be seen as a form of preferential treatment, where management practices benefit a group of animals or herds, creating masked phenotypes. Masked phenotypes could introduce bias in genetic evaluation, not only for cows but also young sires, which can receive biased information from the dam's side, or even for bulls that have daughters with masked records (Burnside and Meyer, 1988; Kuhn et al., 1994, 1999; Tsuruta et al., 2000).

Although the main method of estrus detection in Canada is still visual observation (Van Schyndel et al., 2019), the use of hormonal synchronization has increased over the years (Van Doormaal, 2018). Around $20 \%$ of cows were submitted to timed AI protocols in Canada in 2015 (Van Schyndel et al., 2019). The Canadian Dairy Network (CDN) reported that in 2017, around $30 \%$ of herds had more than $50 \%$ of their breeding done on 1 or $2 \mathrm{~d}$ of the week, suggesting the use of timed AI (Van Doormaal, 2018). This number is significantly higher in the US, where around $87 \%$ of herds make use of hormonal synchronization (Caraviello et al., 2010).

Hormonal synchronization used for timed AI substantially alters the reproductive physiology and endocrinology involved in estrous cycles. Treated animals are set to initiate a new follicle wave, followed by ovulation, giving a higher chance to conceive even for naturally low fertile cows. Therefore, genetically inferior cows will have their performance changed by the hormone protocols and will be more alike cows that naturally show estrus. As genetic programs rely on the collection of accurate phenotypic data, these masked phenotypes could potentially add bias to genetic evaluations (Tsuruta et al., 2000; Bouquet and Juga, 2013). This reproductive practice can also cause changes in the genetic and residual variation when compared with nonsynchronized herds (Goodling et al., 2005).

Information about which herds or cows are on timed $\mathrm{AI}$ is not currently available in most national recording programs. Although phenotypes of synchronized cows might be a source of bias for genetic evaluations, there is only a limited number of studies tackling this problem. Including genetic correlated traits that are not affected by hormonal synchronization in multiple trait models could mitigate the potential bias on the genetic evaluations. Therefore, using simplified simulated Hol- stein populations, this study aimed to: (1) assess the potential effects of timed AI on the estimated genetic parameters of female fertility traits, and (2) propose the inclusion of genetically correlated traits in a multiple trait model as a way to mitigate the potential bias caused by the use of hormones.

\section{MATERIALS AND METHODS}

\section{Ethics Statement}

No animal care committee approval was necessary for the purposes of this study, as it was based entirely on simulated data.

\section{Simulated Populations}

Holstein populations were simulated using a customized Fortran 95 program written by Larry R. Schaeffer at University of Guelph. A starting population of 400 sires and 3,000 dams was established and randomly mated for 3 generations (base population). Subsequently, 17 more generations were used to expand the starting population, resulting in 30,000 cows that were randomly distributed in 200 herds. Five traits were generated per cow, and only one record per cow. Fertility traits were the interval from calving to first service (CTFS) and the interval of first service to conception (FSTC), as these are the most affected traits by hormonal synchronization among currently measured traits. The simulation assumed that all hormonally treated cows get pregnant in the first service. Two conformation traits were also simulated, BCS and angularity score (ANG) both of which had genetic correlations with CTFS and FSTC of 0.40. Last, an uncorrelated trait representing a production index (PI) was included for making breeding decisions. The simulation model included year at calving, herd-year-seasons of birth of the cow, animal additive genetic and residual effects. The assumed parameters were obtained from CDN (Table 1). The results were based on 100 replicates.

\section{Objective 1}

In objective 1 (SIM1), it was assumed that the information about what cows were bred on timed AI was unknown and these cows were included in the data set along with nonsynchronized cows. This mimics what is currently happening in most of the genetic evaluation systems because the reproductive protocol information is mostly unavailable. Given the percentages of herds and cows within herd that were given the hormone protocol, their phenotypes of CTFS and FSTC were changed respectively to 86 (mean of CTFS) and $0 \mathrm{~d}$, as 
Table 1. Covariance matrices for genetic $(\mathbf{G})$, herd-year-season $(\mathbf{H})$, and residual $(\mathbf{R})$ components used in the simulation process ${ }^{1}$

\begin{tabular}{llrrrrr}
\hline Matrix & Trait & CTFS & FSTC & BCS & ANG & PI \\
\hline G & CTFS & 43.72 & 30.66 & -4.10 & 13.70 & 0 \\
& FSTC & 30.66 & 60.89 & -2.80 & 16.50 & 0 \\
& BCS & -4.10 & -2.80 & 2.00 & -0.40 & 0 \\
& ANG & 13.70 & 16.50 & -0.40 & 23.00 & 0 \\
$\mathbf{H}$ & PI & 0 & 0 & 0 & 0 & 25 \\
& CTFS & 258.00 & -65.91 & -3.80 & -0.80 & 0 \\
& FSTC & -65.91 & 80.90 & -2.00 & 3.00 & 0 \\
& BCS & -3.80 & -2.00 & 9.56 & -0.20 & 0 \\
& ANG & -0.80 & 3.00 & -0.20 & 50.00 & 0 \\
$\mathbf{R}$ & PI & 0 & 0 & 0 & 0 & 10 \\
& CTFS & 648.33 & -58.51 & -3.10 & -0.50 & 0 \\
& FSTC & -58.51 & $1,896.94$ & -1.80 & 3.80 & 0 \\
& BCS & -3.10 & -1.80 & 8.00 & -0.80 & 0 \\
& ANG & -0.50 & 3.80 & -0.80 & 96.00 & 0 \\
& PI & 0 & 0 & 0 & 0 & 65 \\
\hline
\end{tabular}

${ }^{1} \mathrm{CTFS}=$ calving to first service in days; FSTC $=$ first service to conception in days; $\mathrm{BCS}=$ body condition score in 1 to 9 scores; $\mathrm{ANG}=$ angularity in 1 to 9 scores; PI = production index.

a result of the hormone treatment. When setting 0 to FSTC it was assumed that all treated cows get pregnant. The data then were analyzed in a multiple trait analysis including just CTFS, FSTC, and PI (BCS and ANG were ignored). Thirteen sets of percentages of herds and cows within herds given the hormone protocol are shown in Table 2. These scenarios were numbered and grouped as: (a) a control scenario where no hormonal synchronization was used, and breeding was based only on natural estrus (S1); (b) a combination of natural heat detection and timed AI (S2-S9); and (c) all cows within herds were synchronized (S10-S12). For scenarios in b, a different proportion of herds were assumed to be using timed AI, with 25, 50, 75, and $100 \%$ of herds randomly selected in the simulated population. In addition to the different proportion of herds, different proportions of cows within each herd were assumed to be on timed AI, with 25 or $50 \%$ of cows randomly chosen within a given herd. For scenarios in c, all cows were considered to be under timed AI within herds, with 25,50 , and $75 \%$ of herds randomly selected to be using timed AI protocols.

According to CDN's report (Van Doormaal, 2018), S6 (or even S10) would be close to what was expected in Canada in 2017, with around $30 \%$ of herds on timed AI.

Four criteria were used to measure the effect of timed AI on the predicted breeding values: (1) correlation between TBV and EBV (accuracy); (2) differences in the mean EBV of top 25, 50, 75, and 100 sires; (3) changes in correlation between TBV and EBV rankings; and (4) changes in the population mean EBV over 20 generations.

\section{Objective 2}

Animals were simulated as in SIM1 and with the same scenarios. However, in objective 2 (SIM2) information on which cows get synchronized was assumed to be known by the genetic evaluation system. Given this, cows that were on timed AI had their CTFS and FSTC treated as missing. Consequently, there were fewer fertility observations than in SIM1, because the masked phenotypes were not included in the analyses. A multiple trait analysis was performed for 5 traits, including BCS and ANG, which are genetically correlated with CTFS and FSTC. As in SIM1, PI was considered as an uncorrelated trait. The same criteria as in SIM1 were

Table 2. Simulated scenarios (S1-S12) considering different proportion of herds and different proportion of cows within herds under hormonal synchronization

\begin{tabular}{|c|c|c|c|c|c|c|c|c|c|c|c|c|}
\hline Proportion & \multicolumn{12}{|c|}{ Scenario } \\
\hline Herds (\%) & 0 & 25 & 50 & 75 & 100 & 25 & 50 & 75 & 100 & 25 & 50 & 75 \\
\hline Overall (\%) & 0 & 6.75 & 12.5 & 18.7 & 25 & 12.5 & 25 & 37.5 & 50 & 25 & 50 & 75 \\
\hline
\end{tabular}


used to quantify the effectiveness of the multiple trait analysis, including highly correlated traits.

\section{Statistical Model}

Breeding values were estimated for all animals (sires, dams, and cows) using the following multitrait animal model:

$$
\mathbf{y}=\mathbf{X b}+\mathbf{Z a}+\mathbf{W h}+\mathbf{e},
$$

where $\mathbf{y}$ is the vector of observations (SIM1 $=$ CTFS, FSTC, and PI; SIM2 = CTFS, FSTC, BCS, ANG, and $\mathrm{PI})$; $\mathbf{b}$ is the vector of fixed effects of cow's birth year; $\mathbf{a}$ is the vector of random additive genetic effects; $\mathbf{h}$ is the vector of random herd-year-season of calving effects; $\mathbf{e}$ is the vector of random residuals; $\mathbf{X}, \mathbf{Z}$, and $\mathbf{W}$ are the model. The covariance matrix was defined as

$$
\mathbf{V}=\operatorname{var}\left[\begin{array}{l}
a \\
h \\
e
\end{array}\right]=\left[\begin{array}{ccc}
\mathbf{G} \otimes \mathbf{A} & 0 & 0 \\
0 & \mathbf{H} \otimes \mathbf{I} & 0 \\
0 & 0 & \mathbf{R} \otimes \mathbf{I}
\end{array}\right],
$$

assuming that $\left[\begin{array}{l}a \\ h \\ e\end{array}\right] \sim N(\mathbf{0}, \mathbf{V})$ for additive genetic $(\mathbf{G})$,

herd-year-season $(\mathbf{H})$, and residual $(\mathbf{R})$ covariance matrices in Table 1, and $\mathbf{A}$ is the additive relationship matrix.

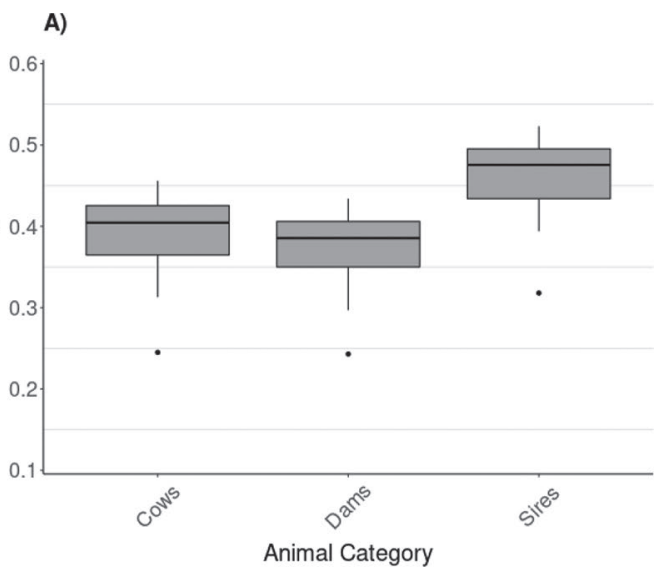
design matrices relating observations in $\mathbf{y}$ to factors in

\section{RESULTS AND DISCUSSION}

\section{Objective 1}

For the SIM1, the accuracy (correlation between TBV and EBV) within each class of animals (cow, dam, and sire) decreased proportionally with the increased use of timed AI. Scenarios in which all cows within herds were on timed AI (S10-S12) had a sharper decrease in accuracy compared with other scenarios. On average $( \pm \mathrm{SD}), \mathrm{EBV}$ of sires were less affected than those of cows and dams, with accuracy (CTFS) averages of 0.46 $\pm 0.06,0.39 \pm 0.06$, and $0.37 \pm 0.05$, respectively (Figure 1A). This was expected because sires have multiple daughters (some masked and some not) contributing to their predicted breeding values, whereas cows were either on or off treatment (Dassonneville et al., 2012). The dot outside of the interquartile range represents scenario S12, being the most affected (low accuracy) regardless of animal categories. When analyzing accuracy by scenarios, it clearly decreased with the increase of synchronized animals (Figure 2A).

The mean EBV of the top (best 25, 50, 75, or 100 sires) approached zero when the number of treated animals increased (Figure 3A). This is likely an effect of the reduction of variance observed by synchronization. When considering the top 25 ranked animals, the mean EBV difference from S1 and S6 was -0.09 , and -0.04 standard deviation (SD) for CTFS, and FSTC, respectively. This difference (S6-S1) slightly decreased when the number of top animals increased, being -0.06 , and -0.02 SD for CTFS and FSTC when considering the top 100 sires. When increasing the proportion of

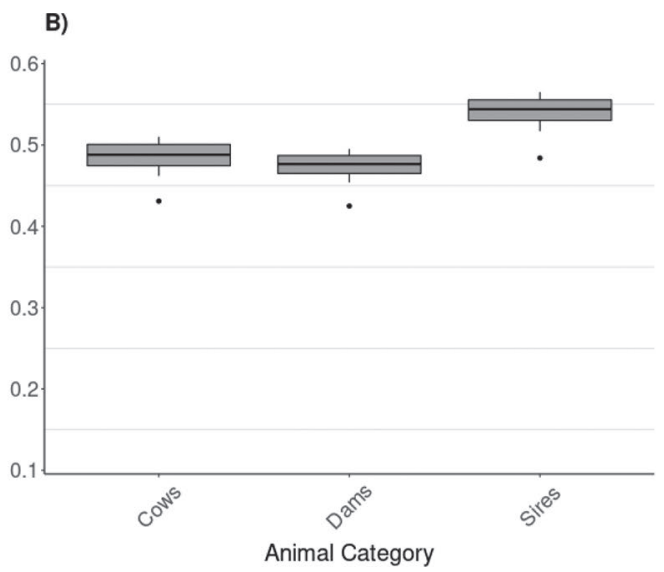

Figure 1. Boxplot of the correlation between true and estimated breeding values (accuracy) of all simulated scenarios per animal category for calving to first service. The gray box represents the interquartile range (IQR), harboring first (Q1) and third (Q3) quartiles, with the median represented by the dark line; the whiskers indicate the variability outside the upper $(\mathrm{Q} 3+1.5 \times \mathrm{IQR})$ and lower $(\mathrm{Q} 1-1.5 \times \mathrm{IQR})$ quartiles; and the dots represent outlier observations. $\mathrm{A}=$ simulated population for objective $1 ; \mathrm{B}=$ simulated population for objective 2 . 

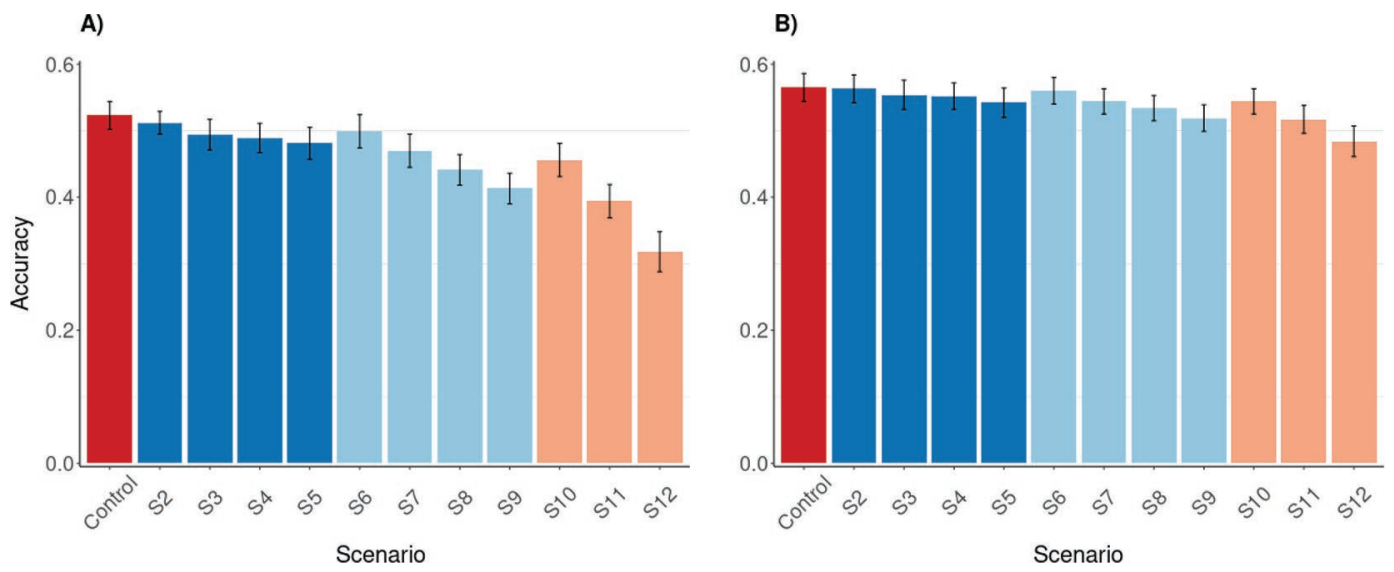

Figure 2. Sires' average accuracy among scenarios (S2-S12) for calving to first service. A = simulated population for objective 1; B = simulated population for objective 2. Figure colors: red $=$ control scenario (no timed AI); dark blue $=25 \%$ of cows on timed AI; light blue $=50 \%$ of cows on timed AI; orange $=$ all cows were under timed AI. The error bars represent mean $\pm \mathrm{SD}$.

treated cow within herds (S10), the difference from the control scenario was -0.23 and -0.14 SD for CTFS when considering top 25 and top 10, respectively.

The sire rank correlation between EBV of simulated scenarios and TBV showed lower correlations in scenarios with more synchronized animals (Figure 4A), varying between 0.67 (S1) to 0.38 (S12) for CTFS and from 0.56 (S1) to 0.21 (S12) for FSTC. In summary, strong sire re-ranking was observed, especially when timed AI was used more intensively. Bias could also exist due to the violation of the assumption that residual variance was not influenced by genetic values, if a preferred group of sires is used for timed AI. These sires would have daughters with more uniform phenotypes than other sires, biasing their comparison.

Because reproductive traits were not considered for selection purposes, and there was no genetic correla-

\section{A)}

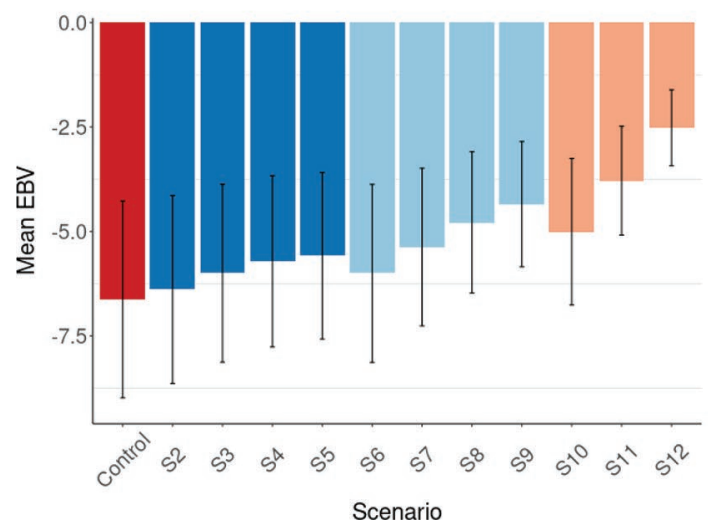

tion between PI and these traits, it was expected a stable trend of mean EBV over generations for both CTFS and FSTC. However, the mean EBV trend of these traits was not linear, being most affected in scenarios that considered more intense use of hormonal synchronization as illustrated in Figure 5A for CTFS. The overall mean CTFS EBV over the last 5 simulated generations ranged from -0.29 (S5) to -1.15 (S10) d. Similar behavior was observed in FSTC. Long-term use of synchronization clearly affected the mean EBV trend of the population for CTFS and FSTC, showing that evaluation for these traits were biased and the bias increased over generations. As already mentioned, CDN estimated that around $30 \%$ of herds were using timed AI in 2017. Based on this estimation, S6 and S10 would be the most realistic scenarios. However, the CDN report (Van Doormaal, 2018) showed an increasing trend

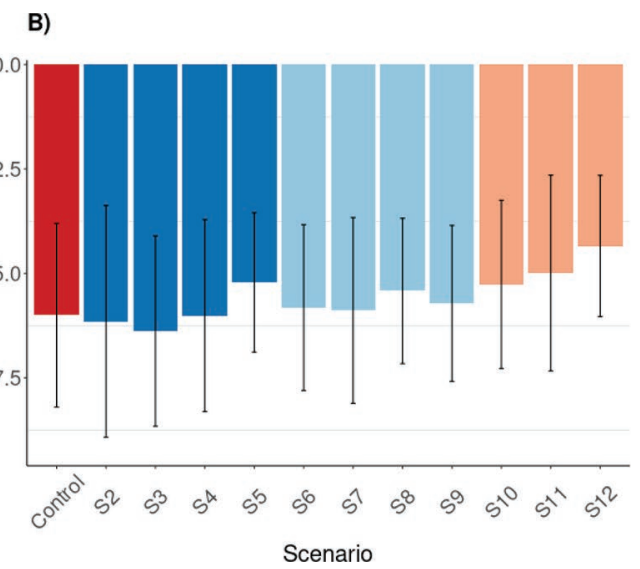

Figure 3. Mean EBV of top 25 sires among scenarios (S2-S12) for calving to first service. A = simulated population for objective 1; B = simulated population for objective 2 . Figure colors: red $=$ control scenario (no timed AI); dark blue $=25 \%$ of cows on timed AI; light blue $=$ $50 \%$ of cows on timed AI; orange $=$ all cows were under timed AI. The error bars represent mean \pm SD. 
A)

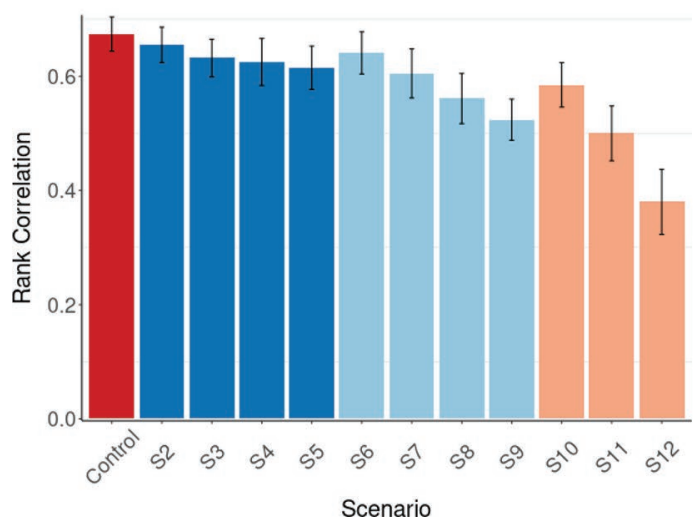

B)

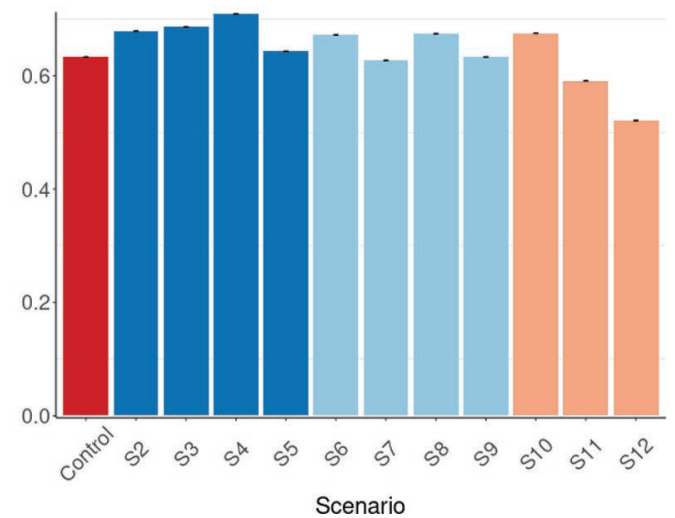

Figure 4. Rank correlation of sires among scenarios (S2-S12) for calving to first service. $\mathrm{A}=$ simulated population for objective 1; $\mathrm{B}=$ simulated population for objective 2. Figure colors: red $=$ control scenario (no timed AI); dark blue $=25 \%$ of cows on timed AI; light blue $=$ $50 \%$ of cows on timed AI; orange = all cows were under timed AI. The error bars represent mean \pm SD.

of the use of timed AI in Canada. Also, the information on what herds and cows are using hormonal synchronization is still not available for breeding programs. So, the range of scenarios in this simulation can help understand the effect of time AI on genetic evaluations in different potential settings.

Issues of bias due to masked phenotypes is also relevant to genomic selection (Dassonneville et al., 2012). The training population used for genomic evaluation relies on animals with both genotype and phenotype information. The inclusion of females in this training population has been proposed in several studies to increase the size of the reference population, and conse- quently, to improve the accuracy of genomic predictions (Plieschke et al., 2016; Uemoto et al., 2017). However, the inclusion of females' records that were masked by hormonal synchronization might introduce bias in the estimation of marker effects, potentially decreasing the accuracy of genomic predictions (Wiggans et al., 2011; Dassonneville et al., 2012; Bouquet and Juga, 2013). This bias would especially affect young animals, whose predictions were based on parent average and SNP effects. Moreover, bulls that had their proofs biased by any preferential treatment could be wrongly ranked, leading to selection mistakes (Gengler et al., 2000; Ducrocq et al., 2003).
A)

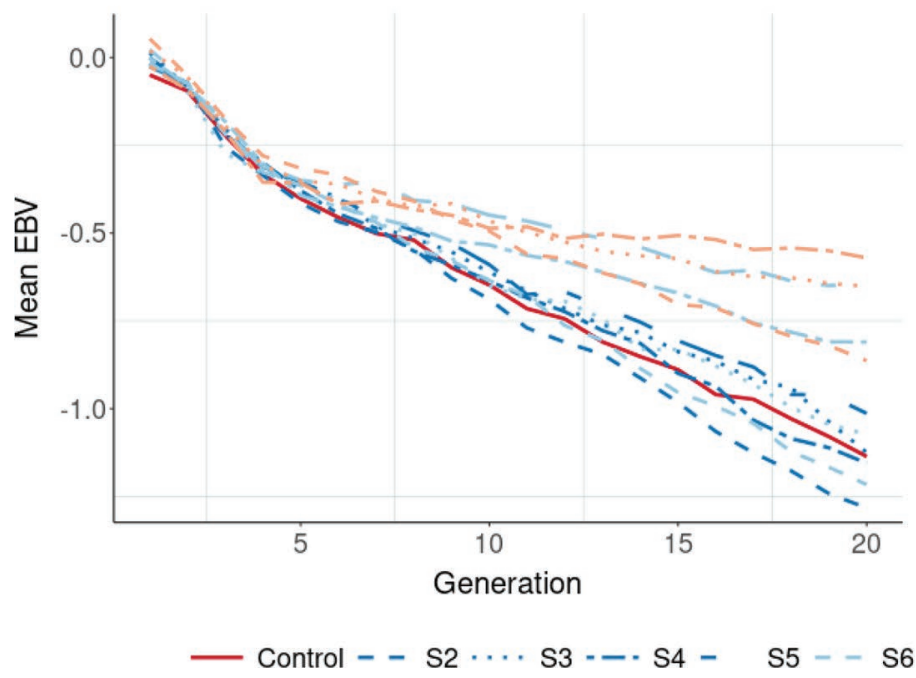

B)

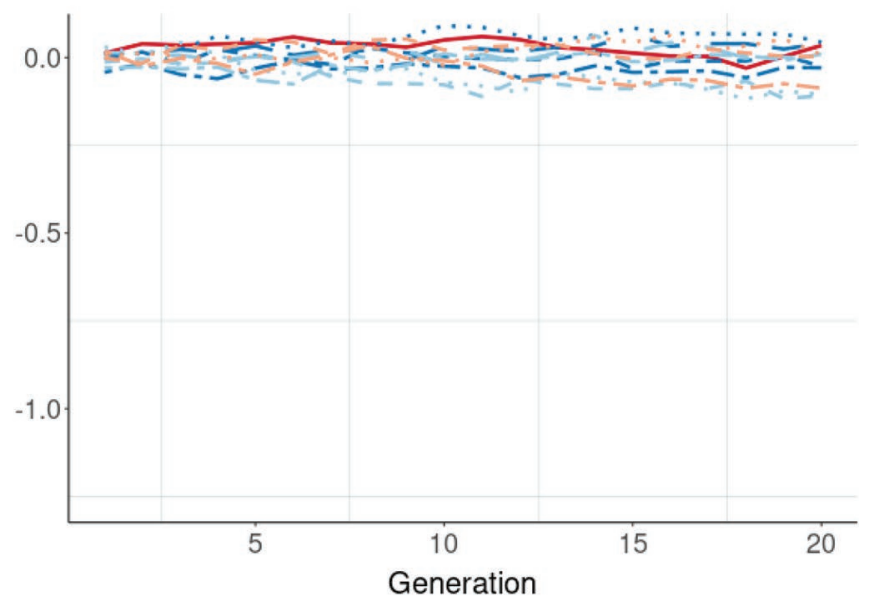

$\mathrm{S} 7-\mathrm{S} 8-\mathrm{S} 9--\mathrm{S} 10 \cdots \mathrm{S} 11-\mathrm{S} 12$

Figure 5. Population mean EBV trend over generations among scenarios (S2-S12) for calving to first service. A $=$ simulated population for

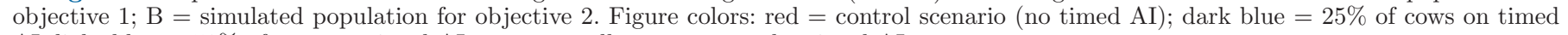
$\mathrm{AI}$; light blue $=50 \%$ of cows on timed AI; orange $=$ all cows were under timed AI. 
Wiggans et al. (2011) proposed a method to adjust genotyped cow traditional evaluations (de-regressed $\mathrm{EBV}$ ) before their inclusion in genomic evaluations. The method relies on the adjustment of the mean and variances of the estimated Mendelian sampling term of genotyped cows and was implemented in the United States in 2010 for Holstein and Jersey breeds. Although the prior adjustment of cow records may provide more accurate genomic predictions, it is still necessary to know which animals were preferentially treated. Even when the proper information is available, another drawback is distinguishing positive Mendelian sampling from bias due to preferential treatment. In 2014, the CDN (now Lactanet) applied a similar method to account for bias of nonrandom usage of highly ranked genomic young bulls, and also for preferential treatment of cows. This adjustment was based on the distribution of the difference between cow evaluations and their pedigree index, which is assumed to follow a normal distribution (Beavers and Van Doormaal, 2014). However, this methodology is currently applied just for somatic cell score, production and type traits, and does not include fertility traits.

The effect of having biased EBV not only affects domestic breeding rograms but also might affect international proofs such as the multiple trait across country evaluations from Interbull. Ducrocq et al. (2003) have argued that the international comparison of dairy bulls can be sensitive to the accuracy and unbiasedness of national evaluations.

Timed AI is a well-established management tool acting on synchronization of follicle growth, regression of the corpus luteum, and ovulation, allowing animals to be inseminated regardless of estrus detection (Colazo and Mapletoft, 2014). Herds with poor estrus detection efficiency are the ones that most benefit from timed $\mathrm{AI}$, which consequently increases the conception rate of these herds. Aside from its management importance, the current investigation has shown that the information on what animals were hormonally treated is crucial for breeding programs.

Apart from the effect on genetic evaluations, public opinion has an increasing role in the dairy industry, being considered the most critical issue in the production system in a Canadian survey in 2016 (Bauman et al., 2016). There is concern about the extensive use of hormone therapies in animal production, which could limit their use in the future (Saint-Dizier and Chastant-Maillard, 2012). Other management tools, such as automated activity monitoring (Løvendahl and Chagunda, 2010), milk progesterone measurement (Saint-Dizier and Chastant-Maillard, 2012), vocalization behavior (Schön et al., 2007), and video inspection systems (Bruyère et al., 2012) have become more reli- able, with potential to further minimize the use of hormonal synchronization for efficient breeding programs. Automated activity monitoring is becoming more popular in Canada, being the most used management technologies on large dairy farms ( $>86$ cows) according to Van Schyndel et al. (2019). Some authors (Neves et al., 2012; Dolecheck et al., 2016) have mentioned that it is possible to maintain comparable reproductive efficiency between estrus-detection-based reproductive protocols and those that heavily rely on timed AI.

Furthermore, the use of hormonal synchronization modifies the interpretation of currently measured traits, and consequently complicates the interpretation of results of genetic analysis. For instance, CTFS aims to measure the time that a cow under natural conditions takes to start a new estrous cycle and show heat. As the use of hormonal synchronization artificially changes the physiology of these cows, the trait no longer should have the same interpretation. Therefore, the continuous use of timed AI could bring light to new traits, such as the conception rate of timed AI cows, which could be used by breeding programs to select animals with better response to the hormonal protocols. But still, it is crucial to emphasize the importance of recording treated cows.

\section{Objective 2}

To overcome bias due to masked phenotypes, a multiple trait analysis was proposed in which the masked phenotypes (for CTFS and FSTC) were treated as missing rather than included in the analysis. In addition, 2 traits (BCS and ANG) not affected by hormonal synchronization and genetically correlated with CTFS and FSTC, were included (SIM2). The concept is similar to including weaning weights of beef calves to evaluate yearling weights where only a fraction of animals is allowed to actually have a yearling weight (Pollak and Quaas, 1981).

As in SIM1, the EBV accuracy of sires were on average less affected than in cows and dams, with mean $( \pm \mathrm{SD})$ values of $0.54 \pm 0.02,0.48 \pm 0.02$, and $0.47 \pm$ 0.02, respectively, for CTFS (Figure 1B). Also following the same pattern as in SIM1, the boxplots in Figure 1B showed S12 as an outlier, with lower accuracy than the other scenarios. Accuracy of sire EBV per scenario is given in Figure 2B. Accuracies were greater than in Figure $2 \mathrm{~A}$ but did decrease as percentage of cows with missing CTFS and FSTC increased, because there was a lower amount of phenotypic information contributing to the calculation of the EBV. Average EBV of the top 25 sires did not differ greatly except when more cows' phenotypes were missing (Figure 3B). Animals were better ranked (closer to TBV ranking) than in the first 
simulation (SIM1; Figure 4B). Finally, the mean EBV trends for CTFS were not affected over time, which should be the case if fertility was not in the PI used to make mating decisions (Figure 5B).

Multiple trait models including genetically correlated traits and treating masked phenotypes as missing worked well in removing bias from EBV for fertility. This approach would not work as well in situations where large percentages of herds and or cows had missing phenotypes due to the hormone protocols. Also, there needs to be a reliable way to identify masked phenotypes. Other, more precise fertility measures need to be adopted, such as automated activity monitors and milk progesterone levels as previously discussed. Only 2 additional traits were included here, but there could be several traits.

This study did not examine repeated records on cows as the cows age. Each cow had only a single record. In reality, cows would have one record for each calving, and a cumulative environmental model would need to be used. For simplicity of demonstration, only single records were needed. No correlation was assumed between the PI and the reproductive traits, allowing for the effect of timed AI to be assessed without the confounding with a negative correlated response on reproductive traits due to selection for production. In addition, for straightforwardness, it was assumed that all hormonally treated cows get pregnant in the first service, making the trait FSTC to be 0 for all timed AI cows.

\section{CONCLUSIONS}

This simulation study showed that genetic evaluations including cow records from the use of timed AI are likely biased, and the amount of bias is proportional to the number of animals on timed AI. Although several strong assumptions were made to illustrate this effect, we conclude that genetic evaluation for reproductive traits could benefit from taking into consideration the presence of masked phenotypic records by hormonal treatment, in terms of accuracy and bias of EBV, and the ultimate ranking of the sires. The inclusion of genetically correlated traits in a multiple trait model was effective in mitigating the bias due to the presence of hormonal synchronized cows, as long as synchronized cows are identified and recorded. However, this is a first study on this topic and further analysis, especially with real data, should be done to validate our findings.

Findings in this study corroborate with the need for reliable phenotypic information in conducting genetic evaluations for selective breeding. The collection of good quality phenotypes (e.g., not masked by hormonal treatment) and recording of the individuals that were hormonally treated appear to be pivotal. The inclusion of correlated indicator traits is a viable modeling strategy. With the increasing use of precision farming technologies that allow to collect a multitude of different phenotypes, more emphasis will have to be placed on the validation of such phenotypes with special focus on bias introduced by hormonal treatment.

\section{ACKNOWLEDGMENTS}

This research was financially supported by Agriculture and Agri-Food Canada, and by additional contributions from Dairy Farmers of Canada, the Canadian Dairy Network and the Canadian Dairy Commission under the Agri-Science Clusters Initiative. As per the research agreement, aside from providing financial support, the funders have no role in the design and conduct of the studies, data collection and analysis, or interpretation of the data. Researchers maintain independence in conducting their studies, own their data, and report the outcomes regardless of the results. The decision to publish the findings rests solely with the researchers. C. Baes acknowledges support from the Natural Sciences and Engineering Research Council of Canada (NSERC) Canada Research Chair (CRC) program. The authors declare that there are no conflicts of interest.

\section{REFERENCES}

Bauman, C. A., H. W. Barkema, J. Dubuc, G. P. Keefe, and D. F. Kelton. 2016. Identifying management and disease priorities of Canadian dairy industry stakeholders. J. Dairy Sci. 99:10194-10203. https://doi.org/10.3168/jds.2016-11057.

Beavers, L., and B. Van Doormaal. 2014. Steps to reduce bias in genetic and genomic evaluations. Accessed Jan. 16, 2020. https:// www.cdn.ca/document.php?id=362.

Bouquet, A., and J. Juga. 2013. Integrating genomic selection into dairy cattle breeding programmes: A review. Animal 7:705-713. https://doi.org/10.1017/S1751731112002248.

Bruyère, P., T. Hétreau, C. Ponsart, J. Gatien, S. Buff, C. Disenhaus, O. Giroud, and P. Guérin. 2012. Can video cameras replace visual estrus detection in dairy cows? Theriogenology 77:525-530. https: //doi.org/10.1016/j.theriogenology.2011.08.027.

Burnside, E. B., and K. Meyer. 1988. Potential impact of bovine somatotropin on dairy sire evaluation. J. Dairy Sci. 71:2210-2219. https://doi.org/10.3168/jds.S0022-0302(88)79795-7.

Butler, W. R. 2003. Energy balance relationships with follicular development, ovulation and fertility in postpartum dairy cows. Livest. Prod. Sci. 83:211-218. https://doi.org/10.1016/S0301 -6226(03)00112-X.

Caraviello, D. Z., K. A. Weigel, P. M. Fricke, M. C. Wiltbank, M. J. Florent, N. B. Cook, K. V. Nordlund, N. R. Zwald, and C. L. Rawson. 2006. Survey of management practices on reproductive performance of dairy cattle on large US commercial farms. J. Dairy Sci. 89:4723-4735. https://doi.org/10.3168/jds.S0022 -0302(06)72522-X.

Colazo, M. G., and R. J. Mapletoft. 2014. A review of current timed-AI (TAI) programs for beef and dairy cattle. Can. Vet. J. 55:772-780.

Dassonneville, R., A. Baur, S. Fritz, D. Boichard, and V. Ducrocq. 2012. Inclusion of cow records in genomic evaluations and impact on bias due to preferential treatment. Genet. Sel. Evol. 44:40. https://doi.org/10.1186/1297-9686-44-40. 
Diskin, M. G., and J. M. Sreenan. 2000. Expression and detection of oestrus in cattle. Reprod. Nutr. Dev. 40:481-491. https://doi.org/ 10.1051/rnd:2000112.

Dolecheck, K. A., W. J. Silvia, G. Heersche Jr., C. L. Wood, K. J. McQuerry, and J. M. Bewley. 2016. A comparison of timed artificial insemination and automated activity monitoring with hormone intervention in 3 commercial dairy herds. J. Dairy Sci. 99:1506-1514. https://doi.org/10.3168/jds.2015-9914.

Ducrocq, V., I. Delaunay, D. Boichard, and S. Mattalia. 2003. A general approach for international genetic evaluations robust to inconsistencies of genetic trends in national evaluations. Interbull Bull. 30:101-111.

Gengler, N., T. Dusseldorf, G. R. Wiggans, J. R. Wright, and T. Druet. 2001. Joint estimation of variances and effects in the US Jersey type evaluation system. Interbull Bull. 26:34-40.

Goodling, R. C. Jr, G. E. Shook, K. A. Weigel, and N. R. Zwald. 2005. The effect of synchronization on genetic parameters of reproductive traits in dairy cattle. J. Dairy Sci. 88:2217-2225.

Jamrozik, J., J. Fatehi, G. J. Kistemaker, and L. R. Schaeffer. 2005. Estimates of genetic parameters for Canadian Holstein female reproduction traits. J. Dairy Sci. 88:2199-2208. https://doi.org/10 .3168/jds.S0022-0302(05)72895-2.

Kerbrat, S., and C. Disenhaus. 2004. A proposition for an updated behavioural characterisation of the oestrus period in dairy cows. Appl. Anim. Behav. Sci. 87:223-238. https://doi.org/10.1016/j .applanim.2003.12.001.

Kuhn, M. T., P. J. Boettcher, and A. E. Freeman. 1994. Potential biases in predicted transmitting abilities of females from preferential treatment. J. Dairy Sci. 77:2428-2437. https://doi.org/10.3168/jds .S0022-0302(94)77185-X.

Kuhn, M. T., A. E. Freeman, and R. L. Fernando. 1999. Approaches investigated to correct for preferential treatment. J. Dairy Sci. 82:181-190. https://doi.org/10.3168/jds.S0022-0302(99)75222-7.

Løvendahl, P., and M. G. G. Chagunda. 2010. On the use of physical activity monitoring for estrus detection in dairy cows. J. Dairy Sci. 93:249-259. https://doi.org/10.3168/jds.2008-1721.

Miglior, F., A. Fleming, F. Malchiodi, L. F. Brito, P. Martin, and C. F. Baes. 2017. A 100-year review: Identification and genetic selection of economically important traits in dairy cattle. J. Dairy Sci. 100:10251-10271. https://doi.org/10.3168/jds.2017-12968.

Neves, R. C., K. E. Leslie, J. S. Walton, and S. J. LeBlanc. 2012. Reproductive performance with an automated activity monitoring system versus a synchronized breeding program. J. Dairy Sci. 95:5683-5693. https://doi.org/10.3168/jds.2011-5264.

Plieschke, L., C. Edel, E. C. G. Pimentel, R. Emmerling, J. Bennewitz, and K.-U. Götz. 2016. Systematic genotyping of groups of cows to improve genomic estimated breeding values of selection candidates. Genet. Sel. Evol. 48:73. https://doi.org/10.1186/s12711 $-016-0250-9$.
Pollak, E. J., and R. L. Quaas. 1981. Monte Carlo study of genetic evaluations using sequentially selected records. J. Anim. Sci. 52:257-264. https://doi.org/10.2527/jas1981.522257x.

Reith, S., and S. Hoy. 2018. Review: Behavioral signs of estrus and the potential of fully automated systems for detection of estrus in dairy cattle. Animal 12:398-407. https://doi.org/10.1017/ S1751731117001975.

Roelofs, J. B. B., F. J. C. M. van Eerdenburg, N. M. Soede, and B. Kemp. 2005. Various behavioral signs of estrous and their relationship with time of ovulation in dairy cattle. Theriogenology 63:1366-1377. https://doi.org/10.1016/j.theriogenology.2004.07 .009 .

Saint-Dizier, M., and S. Chastant-Maillard. 2012. Towards an automated detection of oestrus in dairy cattle. Reprod. Domest. Anim. 47:1056-1061. https://doi.org/10.1111/j.1439-0531.2011.01971.x.

Schön, P. C., K. Hämel, B. Puppe, A. Tuchscherer, W. Kanitz, and G. Manteuffel. 2007. Altered vocalization rate during the estrous cycle in dairy cattle. J. Dairy Sci. 90:202-206. https://doi.org/10 .3168/jds.S0022-0302(07)72621-8.

Silper, B. F., A. M. L. Madureira, L. B. Polsky, S. Soriano, A. F. Sica, J. L. M. Vasconcelos, and R. L. A. Cerri. 2017. Daily lying behavior of lactating Holstein cows during an estrus synchronization protocol and its associations with fertility. J. Dairy Sci. 100:8484-8495. https://doi.org/10.3168/jds.2016-12160.

Tsuruta, S., J. F. Keown, L. D. Van Vleck, and I. Misztal. 2000. Bias in genetic evaluations by records of cows treated with bovine somatotropin. J. Dairy Sci. 83:2650-2656. https://doi.org/10.3168/ jds.S0022-0302(00)75158-7.

Uemoto, Y., T. Osawa, and J. Saburi. 2017. Effect of genotyped cows in the reference population on the genomic evaluation of Holstein cattle. Animal 11:382-393. https://doi.org/10.1017/ S1751731116001762.

Van Doormaal, B. 2018. The fertility challenge. Accessed Jan. 16, 2020. https://www.cdn.ca/document.php?id=493.

Van Schyndel, S. J., C. A. Bauman, O. B. Pascottini, D. L. Renaud, J. Dubuc, and D. F. Kelton. 2019. Reproductive management practices on dairy farms: The Canadian National Dairy Study 2015. J. Dairy Sci. 102:1822-1831. https://doi.org/10.3168/jds.2018-14683.

VanRaden, P. M., A. H. Sanders, M. E. Tooker, R. H. Miller, H. D. Norman, M. T. Kuhn, and G. R. Wiggans. 2004. Development of a national genetic evaluation for cow fertility. J. Dairy Sci. 87:22852292. https://doi.org/10.3168/jds.S0022-0302(04)70049-1.

Wiggans, G. R., T. A. Cooper, P. M. VanRaden, and J. B. Cole. 2011. Technical note: Adjustment of traditional cow evaluations to improve accuracy of genomic predictions. J. Dairy Sci. 94:6188-6193. https://doi.org/10.3168/jds.2011-4481. 